\title{
On Closed Form Expressions for Trigonometric Series and Series Involving Bessel or Struve Functions
}

\author{
S. B. Tričković, M. S. Stankovic and V. N. Aleksis
}

\begin{abstract}
We first consider a summation procedure for some trigonometric series in terms of the Riemann zeta and related functions. In some cases these series can be brought in closed form, which means that the infinite series are represented by finite sums. Afterwards, we show some applications of our results to the summation of series involving Bessel or Struve functions. Further, relying on results from the previous sections, we obtain sums of series involving a Bessel or Struve integral. These series are also represented as series in terms of the Riemann zeta and related functions of reciprocal powers and can be brought in closed form in certain cases as well. By replacing the function appearing in a Bessel and Struve integral with particular functions, we find sums of new series.
\end{abstract}

Keywords: Bessel and Struve functions, Riemann zeta and related functions

AMS subject classification: Primary 33C10, secondary 11M06, 65B10

\section{Closed form expressions for trigonometric series}

At first we consider the sums of the trigonometric series

$$
\begin{aligned}
& S_{1}=S_{1}(s, a, b, p, m, \omega, f(x))=\sum_{n=1}^{\infty} \frac{(s)^{n-1} f((a n-b) x)}{(a n-b)^{2 m+p-1}\left((a n-b)^{2}-\omega^{2}\right)} \\
& S_{2}=S_{2}(s, a, b, p, m, \omega, f(y), g(x))=\sum_{n=1}^{\infty} \frac{(s)^{n-1} f((a n-b) y) g((a n-b) x)}{(a n-b)^{2 m+p-1}\left((a n-b)^{2}-\omega^{2}\right)}(2)
\end{aligned}
$$

where $s, a, b$ are in Table I, $m \in \mathbb{N}_{0}, \omega \in \mathbb{R}$ with $\omega \neq a n-b, p=0$ or $p=1$, and $f$ and $g$ are sin or cos. We evaluate $S_{1}$ and $S_{2}$ as sums in terms of the

S. B. Tričković: Univ. of Niš, Fac. of Civil Eng., Dept. Math., Beogradska 14, 18000 Niš, Rep. of Serbia; sbt@mail.gaf.ni.ac.yu

M. S. Stanković and V. N. Aleksić: Univ. of Niš, Fac. of Envir. Eng., Dept. Math., Carnojevića 10a, 18000 Niš, Rep. of Serbia

ISSN 0232-2064 / \$2.50 C Heldermann Verlag Berlin 
Riemann zeta function $\zeta$ and related functions $\eta, \lambda, \beta$ (see [1] or [8]). The representation of trigonometric series can be stated in the general form (see $[2,5,10])$

$$
\begin{aligned}
& \sum_{n=1}^{\infty} \frac{(s)^{n-1} f((a n-b) x)}{(a n-b)^{p-1}\left((a n-b)^{2}-\omega^{2}\right)} \\
& \quad=\frac{s p(1-b)}{2 \omega^{2}}-\frac{s \pi \sin ^{b-1} \frac{\pi \omega}{2}}{4 \omega^{p} \cos \frac{\pi \omega}{2}} f\left(\omega x-\frac{\pi(s+1)(b+\omega)}{2 a}\right)
\end{aligned}
$$

where $\omega \in \mathbb{R}$ with $\omega \neq a n-b$, and where the parameters $a, b, s, f, p$ and the convergence regions we read from Table IV. We will use the decomposition

$$
\frac{1}{r^{2 m}\left(r^{2}-\omega^{2}\right)}=\frac{1}{\omega^{2 m}\left(r^{2}-\omega^{2}\right)}-\sum_{i=1}^{m} \frac{1}{r^{2 i} \omega^{2 m-2 i+2}} .
$$

Series (1) can be then rewritten as

$$
\begin{aligned}
S_{1}= & \frac{1}{\omega^{2 m}} \sum_{n=1}^{\infty} \frac{(s)^{n-1} f((a n-b) x)}{(a n-b)^{p-1}\left((a n-b)^{2}-\omega^{2}\right)} \\
& -\sum_{i=1}^{m} \frac{1}{\omega^{2 m-2 i+2}} \sum_{n=1}^{\infty} \frac{(s)^{n-1} f((a n-b) x)}{(a n-b)^{2 i+p-1}} .
\end{aligned}
$$

The first sum in $S_{1}$ we evaluate by means of (3) and for the second one we use the closed form formula

$$
\begin{aligned}
& \sum_{n=1}^{\infty} \frac{(s)^{n-1} f((a n-b) x)}{(a n-b)^{2 i+p-1}} \\
& \quad=(-1)^{\frac{2 i+p-1-\delta}{2}} \frac{c \pi}{2(2 i+p-2) !} x^{2 i+p-2}+\sum_{i=0}^{M}(-1)^{i} \frac{F(p-1-\delta)}{(2 i+\delta) !} x^{2 i+\delta}
\end{aligned}
$$

where $M=i-1$ for $p=0$ and $M=i-\delta$ for $p=1$, which is a special case of a closed form formula obtained from

$$
\sum_{n=1}^{\infty} \frac{(s)^{n-1} f((a n-b) x)}{(a n-b)^{\alpha}}=\frac{c \pi}{2 \Gamma(\alpha) f\left(\frac{\pi \alpha}{2}\right)} x^{\alpha-1}+\sum_{i=0}^{\infty}(-1)^{i} \frac{F(\alpha-2 i-\delta)}{(2 i+\delta) !} x^{2 i+\delta}
$$

where $\alpha \in \mathbb{R}^{+}$and all relevant parameters are given in Table I (see [7]). The closed form cases are given in Table II, and they ensue because functions $F$ 
take the value zero at certain points (see [7]).

So we have

$$
\begin{aligned}
S_{1}= & \frac{s p(1-b)}{2 \omega^{2 m+2}}-\frac{s \pi \sin ^{b-1} \frac{\pi \omega}{2}}{4 \omega^{2 m+p} \cos \frac{\pi \omega}{2}} f\left(\omega x-\frac{\pi(s+1)(b+\omega)}{2 a}\right) \\
& +\frac{c \pi}{2} \sum_{i=1}^{m}(-1)^{i+p} \frac{x^{2 i+p-2}}{\omega^{2 m-2 i+2}(2 i+p-2) !} \\
& -\sum_{i=1}^{m} \sum_{k=0}^{L}(-1)^{k} \frac{F(2 i-2 k+p-1-\delta)}{\omega^{2 m-2 i+2}(2 k+\delta) !} x^{2 k+\delta}
\end{aligned}
$$

where $\omega \in \mathbb{R}$ with $\omega \neq a n-b, f=\left\{\begin{array}{l}\sin \\ \cos \end{array}\right\} \delta=\left\{\begin{array}{l}1 \\ 0\end{array}\right\}, L=i-1+p(1-\delta)$ and the rest of the parameters are given in Table IV.

Note that when we let $\omega \rightarrow 0$ in formulas (3) and (6), we obtain the known formula (5). For instance, setting $s=1, a=1, b=0, p=1, f=\cos$ in formula (3), we come to the formula (see [3: p. 243/Formula (17.3.9)])

$$
\sum_{n=1}^{\infty} \frac{\cos n x}{n^{2}-\omega^{2}}=\frac{1}{2 \omega^{2}}-\frac{\pi \cos (\omega x-\omega \pi)}{2 \omega \sin \omega \pi}
$$

Taking limiting value when $\omega \rightarrow 0$, we get

$$
\sum_{n=1}^{\infty} \frac{\cos n x}{n^{2}}=-\frac{\pi x}{2}+\frac{\pi^{2}}{6}+\frac{x^{2}}{4} .
$$


When we choose $a=1, b=0, s=1, \alpha=2, f=\cos$ in formula (5), the rest of the parameters are $\delta=0, c=1, M=1, F=\zeta$, so we obtain

$$
\sum_{n=1}^{\infty} \frac{\cos n x}{n^{2}}=-\frac{\pi x}{2}+\zeta(2)-\frac{\zeta(0)}{2} x^{2}
$$

This is just what we have got letting $\omega \rightarrow 0$ in formula (7), since it is known that $\zeta(2)=\frac{\pi^{2}}{6}$ and the value $\zeta(0)=-\frac{1}{2}$ we find on the basis of an analytical extension of the function $\zeta$ to the whole complex plane (except for $z=1$, where it has a pole, see [4]).

Now we present a formula comprising sixteen formulas:

$$
\begin{aligned}
& \sum_{n=1}^{\infty} \frac{(s)^{n-1} f((a n-b) y) g((a n-b) x)}{(a n-b)^{p-1}\left((a n-b)^{2}-\omega^{2}\right)} \\
& \quad=g(0) \frac{s p(1-b)}{2 \omega^{2}}-\frac{s \pi \sin ^{b-1} \frac{\pi \omega}{2}}{4 \omega^{p} \cos \frac{\pi \omega}{2}} f\left(\omega y-\frac{\pi(s+1)(b+\omega)}{2 a}\right) g(\omega x)
\end{aligned}
$$

where $\omega \in \mathbb{R}$ with $\omega \neq a n-b$, and where the parameters $a, b, s$ and the convergence regions are in Table III. The functions $f$ and $g$ take values sin or cos and the parameter $p$ we choose so that

$$
p=\left\{\begin{array}{ll}
0 & \text { if } f \neq g \\
1 & \text { if } f=g
\end{array} \text { for } F=\zeta, \eta, \lambda \quad \text { and } \quad p=\left\{\begin{array}{ll}
1 & \text { if } f \neq g \\
0 & \text { if } f=g
\end{array} \text { for } F=\beta\right.\right.
$$

Each of these sixteen formulas is obtained by using the trigonometric identities

$$
\sin (a n-b) y \cos (a n-b) x=\frac{1}{2}(\sin (a n-b)(y-x)+\sin (a n-b)(y+x))
$$

and then applying (3) to both obtained series. Substituting (4) into series (2) we get

$$
\begin{aligned}
S_{2}= & \frac{1}{\omega^{2 m}} \sum_{n=1}^{\infty} \frac{(s)^{n-1} f((a n-b) y) g((a n-b) x)}{(a n-b)^{p-1}\left((a n-b)^{2}-\omega^{2}\right)} \\
& -\sum_{i=1}^{m} \sum_{n=1}^{\infty} \frac{(s)^{n-1} f((a n-b) y) g((a n-b) x)}{\omega^{2 m-2 i+2}(a n-b)^{2 i+p-1}} .
\end{aligned}
$$

We use (8) for the first sum, and for the second one we need the closed form 
formula (see [8])

$$
\begin{aligned}
\sum_{n=1}^{\infty} & \frac{(s)^{n-1} f((a n-b) y) g((a n-b) x)}{(a n-b)^{2 m+p-1}} \\
= & \frac{c \pi}{2} \sum_{i=0}^{m-1-d \delta}(-1)^{m-1+d-d \delta} \frac{x^{2 i+\delta} y^{2 m-2 i-\delta+p-2}}{(2 i+\delta) !(2 m-2 i-\delta+p-2) !} \\
& +\sum_{i=0}^{m-1-d \delta} \sum_{j=0}^{M}(-1)^{i+j} \frac{F(2 m-2 i-2 j-\delta+p-1-t) x^{2 i+\delta} y^{2 j+t}}{(2 i+\delta) !(2 j+t) !} \\
& +\frac{(b-1) s d(-1)^{m-\delta} x^{2 m-\delta}}{2(2 m-\delta) !}
\end{aligned}
$$

where $m \in \mathbb{N}, g=\left\{\begin{array}{l}\sin \\ \cos \end{array}\right\} \delta=\left\{\begin{array}{l}1 \\ 0\end{array}\right\}$ and, independently of that, $f=\left\{\begin{array}{l}\sin \\ \cos \end{array}\right\} t=$ $\left\{\begin{array}{l}1 \\ 0\end{array}\right\}$. The parameter $p$ we choose as in (8), as far as $d$ is concerned it depends on $f$ and we treat it as parameter $p$ in Table IV. The rest of the parameters are given in Table III. So we finally come to the formula

$$
\begin{aligned}
S_{2}= & \frac{g(0) s p(1-b)}{2 \omega^{2 m+2}} \\
& -\frac{s \pi \sin ^{b-1} \frac{\pi \omega}{2}}{4 \omega^{2 m+p} \cos \frac{\pi \omega}{2}} f\left(\omega y-\frac{\pi(s+1)(b+\omega)}{2 a}\right) g(\omega x) \\
& +\frac{s d(1-b)}{2} \sum_{i=1}^{m}(-1)^{i-\delta} \frac{x^{2 i-\delta}}{\omega^{2 m+2-2 i}(2 i-\delta) !} \\
& +\frac{c \pi}{2} \sum_{i=1}^{m} \sum_{k=0}^{i-1-d \delta}(-1)^{i-d-d \delta} \frac{x^{2 k+\delta} y^{2 i-2 k-\delta+p-2}}{\omega^{2 m-2 i+2}(2 k+\delta) !(2 i-2 k-\delta+p-2) !} \\
& -\sum_{i=1}^{m} \sum_{k=0}^{i-1-d \delta} \sum_{j=0}^{M}(-1)^{k+j} \frac{F(2 i-2 k-2 j-\delta-t+p-1) x^{2 k+\delta} y^{2 j+t}}{\omega^{2 m-2 i+2}(2 k+\delta) !(2 j+t) !}
\end{aligned}
$$

where $\omega \in \mathbb{R}$ with $\omega \neq$ an $-b, g=\left\{\begin{array}{l}\sin \\ \cos \end{array}\right\} \delta=\left\{\begin{array}{l}1 \\ 0\end{array}\right\}$ and, independently of that, $f=\left\{\begin{array}{c}\sin \\ \cos \end{array}\right\} t=\left\{\begin{array}{l}1 \\ 0\end{array}\right\}$. Here $M=i-k-1+(-1)^{\delta}(1-p) d$ and the parameter $p$ we choose in the same way as for (8). All other relevant parameters are listed in Table III, except for $d$ which is chosen in the same manner as for (9).

As we have obtained formula (5) by letting $\omega \rightarrow 0$ in (3) and (6), we can similarly come to formula (9) by taking limiting values of formulas (8) and (10) when $\omega \rightarrow 0$. 


\section{Series involving Bessel or Struve functions}

We illustrate here the application of the obtained results to the summation of series involving Bessel or Struve functions and of series containing the product of Bessel and trigonometric function. Both series have coefficients of the same type as in (1) and (2).

2.1 The obtained formulas given by (6) can be applied to the summation of some series involving Bessel or Struve functions [9]. For example, let us consider the series

$$
S_{3}=S_{3}\left(m, \nu, \mu, \varphi_{\nu}(x)\right)=\sum_{n=1}^{\infty} \frac{\varphi_{\nu}(n x)}{n^{2 m-\mu}\left(n^{2}-\omega^{2}\right)}
$$

where $\varphi_{\nu}$ denote $J_{\nu}$ or $\mathbf{H}_{\nu}$, namely the Bessel or Struve function of the first kind and of order $\nu \in \mathbb{R}, n \neq \omega \in \mathbb{R}$ and $\mu \in \mathbb{R}$ with $\mu+\nu=\left\{\begin{array}{l}0 \\ 1\end{array}\right\}$. Using the well-known integral representation of Bessel/Struve functions

$$
\varphi_{\nu}(z)=\frac{2\left(\frac{z}{2}\right)^{\nu}}{\Gamma\left(\frac{1}{2}\right) \Gamma\left(\nu+\frac{1}{2}\right)} \int_{0}^{\frac{\pi}{2}} \sin ^{2 \nu} \theta g(z \cos \theta) d \theta
$$

where $\operatorname{Re} \nu>-\frac{1}{2}, \varphi=\left\{\begin{array}{c}J \\ H\end{array}\right\} g=\left\{\begin{array}{c}\cos \\ \text { sin }\end{array}\right\}$ and, interchanging the order of summation and integration, the series $S_{3}$ takes the form

$$
S_{3}=\frac{2\left(\frac{x}{2}\right)^{\nu}}{\Gamma\left(\frac{1}{2}\right) \Gamma\left(\nu+\frac{1}{2}\right)} \int_{0}^{\frac{\pi}{2}} \sin ^{2 \nu} \theta \sum_{n=1}^{\infty} \frac{g(n x \cos \theta)}{n^{2 m-\mu-\nu}\left(n^{2}-\omega^{2}\right)} d \theta .
$$

The obtained sum is of type (6), with $x \cos \theta$ instead of $x \quad(0<x<2 \pi)$ and for $\mu+\nu=\left\{\begin{array}{l}0 \\ 1\end{array}\right\} g=\left\{\begin{array}{c}\cos \\ \sin \end{array}\right\}$. The further procedure leads to the integrals

$$
\int_{0}^{\frac{\pi}{2}} \sin ^{\mu-1} x \cos ^{\nu-1} x d x=\frac{1}{2} \mathrm{~B}\left(\frac{\mu}{2}, \frac{\nu}{2}\right) \quad(\operatorname{Re} \mu>0, \operatorname{Re} \nu>0) .
$$

Finally, the demanded sum is

$$
\begin{aligned}
S_{3}= & \frac{\left(\frac{x}{2}\right)^{\nu}}{\omega^{2 m+2}}\left(\frac{1-\mu-\nu}{2 \Gamma(\nu+1)}\right. \\
& +\frac{\sqrt{\pi}}{2} \sum_{i=1}^{m}(-1)^{i+1-\mu-\nu} \frac{\Gamma\left(i-\frac{\mu+\nu}{2}\right) \omega^{2 i}}{(2 i-\mu-\nu-1) ! \Gamma\left(i+\frac{\nu-\mu+1}{2}\right)} x^{2 i-\mu-\nu-1} \\
& \left.-\frac{1}{\sqrt{\pi}} \sum_{i=1}^{m} \sum_{k=0}^{L}(-1)^{k} \frac{\omega^{2 i} \zeta(2 i-2 k-\mu-\nu-\delta) \Gamma\left(k+\frac{\delta+1}{2}\right)}{(2 k+\delta) ! \Gamma\left(\nu+k+1+\frac{\delta}{2}\right)} x^{2 k+\delta}\right) \\
& -\frac{\pi}{2 \omega^{2 m+1-\mu} \sin \pi \omega}\left((-1)^{\delta} g(\pi \omega) J_{\nu}(\omega x)+\bar{g}(\pi \omega) \mathbf{H}_{\nu}(\omega x)\right)
\end{aligned}
$$


where $\operatorname{Re} \nu>-\frac{1}{2}, \varphi=\left\{\begin{array}{c}J \\ H\end{array}\right\} g=\left\{\begin{array}{c}\cos \\ \sin \end{array}\right\} \delta=\left\{\begin{array}{l}0 \\ 1\end{array}\right\} \mu+\nu=\left\{\begin{array}{c}0 \\ 1\end{array}\right\}$ and $L=i-1+$ $(1-\mu-\nu)(1-\delta)$.

2.2 We make use of the result given by (8) and apply it to the summation of series containing the product of Bessel or Struve and trigonometric functions:

$$
\begin{aligned}
S_{4} & =S_{f}\left(a, b, s, \mu, \nu, \varphi_{\nu}(x), f(x)\right) \\
& =\sum_{n=1}^{\infty} \frac{(s)^{n-1}(a n-b)^{\mu}}{(a n-b)^{2}-\omega^{2}} \varphi_{\nu}((a n-b) x) f((a n-b) y)
\end{aligned}
$$

where $\varphi_{\nu}$ denote $J_{\nu}$ or $\mathbf{H}_{\nu}$, namely the Bessel or Struve function of the first kind and of order $\nu \in \mathbb{R}, f$ is sin or $\cos , \mu, \omega \in R$ with $\omega \neq a n-b$ and $a, b, s$ are like those in Table I. By applying representation (11) and interchanging the order of summation and integration, the preceding series can be rewritten as

$$
S_{4}=\frac{2\left(\frac{x}{2}\right)^{\nu}}{\Gamma\left(\frac{1}{2}\right) \Gamma\left(\nu+\frac{1}{2}\right)} \int_{0}^{\frac{\pi}{2}} \sin ^{2 \nu} \theta \sum_{n=1}^{\infty} \frac{(s)^{n-1}(a n-b)^{\mu+\nu}}{(a n-b)^{2}-\omega^{2}} g_{n} f_{n} d \theta
$$

where $g_{n}=g((a n-b) x \cos \theta)$ and $f_{n}=f((a n-b) y)$. This sum is given by (8), with $x \cos \theta$ instead of $x$, and for $\mu+\nu=1-p$, with $p$ in Table V. We shall state now the final result as

$$
\begin{aligned}
S_{4}= & \frac{g(0) s(1-\mu-\nu)(1-b)}{2 \omega^{2} \Gamma(\nu+1)}\left(\frac{x}{2}\right)^{\nu} \\
& -\frac{s \pi \sin ^{b-1} \frac{\pi \omega}{2}}{4 \omega^{1-\mu} \cos \frac{\pi \omega}{2}} f\left(\omega y-\frac{\pi(s+1)(b+\omega)}{2 a}\right) \varphi_{\nu}(\omega x)
\end{aligned}
$$

where $\operatorname{Re} \nu>-\frac{1}{2}, \varphi=\left\{\begin{array}{c}J \\ H\end{array}\right\} g=\left\{\begin{array}{c}\cos \\ \text { sin }\end{array}\right\} \mu+\nu=1-p$, with $p$ and the rest of the parameters in Table $\mathrm{V}$.

Note that the summation of series of type (1) and (2) has not been considered in the literature, except for some particular cases. More precisely, series (1) for $m=0$, and these sums one can look up in [2, 5, 10]. Our general formula (3) includes each of them. As far as we know, in the literature there are no corresponding particular cases relating to the new formulas (6), (8) and (10).

The obtained general formula (12) includes four particular cases cited in [6: p. 683]:

$$
\begin{aligned}
& \sum_{k=1}^{\infty} \frac{1}{k^{2}-a^{2}}\left\{\begin{array}{c}
k \sin k b \\
\cos k b
\end{array}\right\} J_{0}(k x) \\
& \quad= \pm \frac{\pi \operatorname{cosec} a \pi}{2 a}\left\{\begin{array}{c}
a \sin a(\pi-b) \\
\cos a(\pi-b)
\end{array}\right\} J_{0}(a x)+\frac{1}{2 a^{2}}\left\{\begin{array}{l}
0 \\
1
\end{array}\right\}
\end{aligned}
$$


where $0<b \pm x<2 \pi$ and

$$
\begin{aligned}
\sum_{k=1}^{\infty}( \pm 1)^{k} \frac{k^{-\nu}}{k^{2}-a^{2}} \cos k x J_{\nu}(k x) & \\
\quad= & \frac{2^{-\nu-1} x^{\nu}}{a^{2} \Gamma(\nu+1)}-\frac{\pi \operatorname{cosec} a \pi}{2 a^{\nu+1}}\left\{\begin{array}{c}
\cos a(x-\pi) \\
\cos a x
\end{array}\right\} J_{\nu}(a x)
\end{aligned}
$$

where $\operatorname{Re} \nu>\frac{-(4 \mp 1)}{2}$ and $0<x<(3 \pm 1) \frac{\pi}{4}$.

\section{Some series over Bessel or Struve integrals}

Relying on previous results we can further expand our considerations. Following procedures similar to those in Section 2 we first find summation formulas for the series

$$
\begin{aligned}
\mathcal{F} & =\sum_{n=1}^{\infty} \frac{(s)^{n-1} F_{\nu}((a n-b) x)}{(a n-b)^{\alpha}} \\
\mathcal{F}_{\omega} & =\sum_{n=1}^{\infty} \frac{(s)^{n-1}(a n-b)^{\mu} F_{\nu}((a n-b) x)}{(a n-b)^{2 m}\left((a n-b)^{2}-\omega^{2}\right)}
\end{aligned}
$$

where $a=\left\{\begin{array}{l}1 \\ 2\end{array}\right\} b=\left\{\begin{array}{l}0 \\ 1\end{array}\right\}, s= \pm 1, \alpha, \mu, \nu, \omega \in \mathbb{R}$ with $\omega \neq a n-b, m \in \mathbb{N}$ and $F_{\nu}=\left\{\begin{array}{c}J_{\nu} \\ \mathbf{H}_{\nu}\end{array}\right\}\left(J_{\nu}\right.$ and $\mathbf{H}_{\nu}$ are Bessel and Struve functions of the first kind and order $\nu)$. So we find

$$
\begin{aligned}
\mathcal{F}= & \frac{c \pi\left(\frac{x}{2}\right)^{\alpha-1}}{2 \Gamma\left(\frac{\alpha-\nu+1}{2}\right) \Gamma\left(\frac{\alpha+\nu+1}{2}\right) f\left(\frac{\pi(\alpha-\nu)}{2}\right)} \\
& +\sum_{i=0}^{\infty}(-1)^{i} \frac{F(\alpha-\nu-2 i-\delta)\left(\frac{x}{2}\right)^{\nu+2 i+\delta}}{\Gamma\left(i+1+\frac{\delta}{2}\right) \Gamma\left(\nu+i+1+\frac{\delta}{2}\right)}
\end{aligned}
$$

where $\alpha, \nu \in \mathbb{R}$ with $\alpha>0$ and $\alpha>\nu>-\frac{1}{2}, F_{\nu}=\left\{\begin{array}{c}J_{\nu} \\ \mathbf{H}_{\nu}\end{array}\right\} f=\left\{\begin{array}{c}\cos \\ \sin \end{array}\right\} \delta=\left\{\begin{array}{l}0 \\ 1\end{array}\right\}$, but $a, b, s, c, F$ and the corresponding convergence regions are given in Table $\mathrm{V}$. When $f=\left\{\begin{array}{c}\cos \\ \sin \end{array}\right\} \alpha-\nu=\left\{\begin{array}{c}2 k+1 \\ 2 k\end{array}\right\}$ with $k \in \mathbb{N}_{0}$, one should work with limiting values or with principal values of gamma functions. We note that even in the case $\alpha-\nu=1$ formula (15) is correct. Truncation of the second series in (15), due to the vanishing of $F$ functions, gives rise to all closed form cases (see Table VI). 
Further, we find

$$
\begin{aligned}
\mathcal{F}_{\omega}= & \frac{\left(\frac{x}{2}\right)^{\nu}}{\omega^{2 m+2}}\left[\frac{s(1-\mu-\nu)}{2 \Gamma(\nu+1)}(1-b)+\frac{c \sqrt{\pi}}{2} \sum_{i=1}^{m} A_{i}-\frac{1}{\sqrt{\pi}} \sum_{i=1}^{m} \sum_{k=0}^{M} B_{i k}\right] \\
& -\frac{s \pi \omega^{\mu-2 m-1} \sin ^{b-1} \frac{\pi \omega}{2}}{4 \cos \frac{\pi \omega}{2}} \\
& \times\left[(-1)^{\delta} f\left(\frac{\pi(\omega+b)(s+1)}{2 a}\right) J_{\nu}(\omega x)+\bar{f}\left(\frac{\pi(\omega+b)(s+1)}{2 a}\right) \mathbf{H}_{\nu}(\omega x)\right]
\end{aligned}
$$

where, for the sake of brevity, we have introduced denotations

$$
\begin{aligned}
A_{i} & =(-1)^{i+1-\mu-\nu} \frac{\Gamma\left(i+\frac{\mu+\nu}{2}\right) \Gamma\left(i-\frac{\mu+\nu}{2}\right) \omega^{2 i}}{(2 i-\mu-\nu-1) ! \Gamma\left(i+\frac{\nu-\mu+1}{2}\right)} x^{2 i-\mu-\nu-1} \\
B_{i k} & =(-1)^{k} \frac{F(2 i-2 k-\mu-\nu-\delta) \Gamma\left(k+\frac{\delta+1}{2}\right) \omega^{2 i} x^{2 k+\delta}}{(2 k+\delta) ! \Gamma\left(\nu+k+1+\frac{\delta}{2}\right)}
\end{aligned}
$$

where $\operatorname{Re} \nu>-\frac{1}{2}, F_{\nu}=\left\{\begin{array}{c}J_{\nu} \\ \mathbf{H}_{\nu}\end{array}\right\} f=\left\{\begin{array}{c}\cos \\ \sin \end{array}\right\} \bar{f}=\left\{\begin{array}{c}\sin \\ \cos \end{array}\right\} \delta=\left\{\begin{array}{c}0 \\ 1\end{array}\right\}, M=i-1+(1-$ $\mu-\nu)(1-\delta)$ and the rest of the parameters is in Table $\mathrm{V}$.

Now we present a procedure providing summation formulas for the series

$$
\begin{aligned}
& I_{1}=\sum_{n=1}^{\infty} \frac{(s)^{n-1} D_{\nu}((a n-b) x)}{(a n-b)^{\alpha}} \\
& I_{2}=\sum_{n=1}^{\infty} \frac{(s)^{n-1}(a n-b)^{\mu} D_{\nu}((a n-b) x)}{(a n-b)^{2}-\omega^{2}} \\
& I_{3}=\sum_{n=1}^{\infty} \frac{(s)^{n-1}(a n-b)^{\mu} D_{\nu}((a n-b) x)}{(a n-b)^{2 m}\left((a n-b)^{2}-\omega^{2}\right)}
\end{aligned}
$$

where $a=\left\{\begin{array}{l}1 \\ 2\end{array}\right\} b=\left\{\begin{array}{l}0 \\ 1\end{array}\right\}, s= \pm 1, \alpha, \mu, \nu, \omega \in \mathbb{R}$ with $\omega \neq$ an $-b, m \in \mathbb{N}$, and $D_{\nu}(x)=\left\{\begin{array}{l}B_{\nu}(x) \\ S_{\nu}(x)\end{array}\right\}$. Here $B_{\nu}$ and $S_{\nu}$ are Bessel and Struve integrals, 
respectively, defined by

$$
B_{\nu}(x)=\int_{0}^{1} J_{\nu}(x y) \varphi(y) d y \quad \text { and } \quad S_{\nu}(x)=\int_{0}^{1} \mathbf{H}_{\nu}(x y) \varphi(y) d y .
$$

We will obtain results similar to those for series (13) and (14) simply by setting there integrals (20) instead of Bessel or Struve functions. The representation of series (17) - (19) is again based on the representation of corresponding series involving Bessel or Struve functions in terms of the Riemann zeta function $\zeta$ and related functions of reciprocal powers $\eta, \lambda, \beta$.

3.1 In order to find the sum of series (17) we first replace the integral of the form $D_{\nu}(x)$, then interchanging the order of summation and integration, we obtain

$$
I_{1}=\int_{0}^{1}\left(\sum_{n=1}^{\infty} \frac{(s)^{n-1} F_{\nu}((a n-b) x y)}{(a n-b)^{\alpha}}\right) \varphi(y) d y \quad\left(\alpha \in \mathbb{R}^{+}\right) .
$$

We further use formula (15), taking $x y$ instead of $x$. Note that the boundaries for $x y$ are the same as those for $x$ because $0 \leq y \leq 1$. Hence the final formula for the summation of series (17) is

$$
\begin{aligned}
I_{1}= & \frac{c \pi x^{\alpha-1}}{2^{\alpha} \Gamma\left(\frac{\alpha-\nu+1}{2}\right) \Gamma\left(\frac{\alpha+\nu+1}{2}\right) f\left(\frac{\pi(\alpha-\nu)}{2}\right)} \int_{0}^{1} y^{\alpha-1} \varphi(y) d y \\
& +\sum_{i=0}^{\infty}(-1)^{i} \frac{F(\alpha-\nu-2 i-\delta) x^{\nu+2 i+\delta}}{2^{\nu+2 i+\delta} \Gamma\left(i+1+\frac{\delta}{2}\right) \Gamma\left(\nu+i+1+\frac{\delta}{2}\right)} \int_{0}^{1} \varphi(y) y^{\nu+2 i+\delta} d y
\end{aligned}
$$

where $D_{\nu}=\left\{\begin{array}{l}B_{\nu} \\ S_{\nu}\end{array}\right\} f=\left\{\begin{array}{c}\cos \\ \sin \end{array}\right\} \delta=\left\{\begin{array}{l}0 \\ 1\end{array}\right\}$ and the rest of parameters can be found in Table $\mathrm{V}$.

3.2 Concerning the sum of series (18), we will apply a similar procedure. We first substitute the integral of the form $D_{\nu}(x)$, then interchange the order of summation and integration. Afterwards we use formula (16), where we set $m=0$. That yields for series (18) the summation formula

$$
I_{2}=\frac{\left(\frac{x}{2}\right)^{\nu}}{\omega^{2}} K(1-b)-\frac{s \pi \omega^{\mu-1} \sin ^{b-1} \frac{\pi \omega}{2}}{4 \cos \frac{\pi \omega}{2}} P
$$

where $\omega \in \mathbb{R}$ with $\omega \neq a n-b$. For the sake of simplicity, we have put

$$
\begin{aligned}
K & =\frac{s(1-\mu-\nu)}{2 \Gamma(\nu+1)} \int_{0}^{1} \varphi(y) y^{\nu} d y \\
P & =(-1)^{\delta} f\left(\frac{\pi(\omega+b)(s+1)}{2 a}\right) B_{\nu}(\omega x)+\bar{f}\left(\frac{\pi(\omega+b)(s+1)}{2 a}\right) S_{\nu}(\omega x) .
\end{aligned}
$$


Here $D_{\nu}(x)=\left\{\begin{array}{c}B_{\nu}(x) \\ S_{\nu}(x)\end{array}\right\} f=\left\{\begin{array}{c}\cos \\ \sin \end{array}\right\} \bar{f}=\left\{\begin{array}{c}\sin \\ \cos \end{array}\right\} \delta=\left\{\begin{array}{l}0 \\ 1\end{array}\right\}$. The other parameters we read from Table V. Particular cases 1 - 8 of (22) are listed in the Appendix.

3.3 Following a similar procedure as that for obtaining (22), by using (16) for $m \geq 1$, we find the sum of series (19) as

$$
\begin{aligned}
I_{3}= & \frac{\left(\frac{x}{2}\right)^{\nu}}{\omega^{2 m+2}}\left(K(1-b)+\frac{c \sqrt{\pi}}{2} \sum_{i=1}^{m} W_{i}-\frac{1}{\sqrt{\pi}} \sum_{i=1}^{m} \sum_{k=0}^{M} Z_{i k}\right) \\
& -\frac{s \pi \omega^{\mu-2 m-1} \sin ^{b-1} \frac{\pi \omega}{2}}{4 \cos \frac{\pi \omega}{2}} P
\end{aligned}
$$

where

$$
W_{i}=A_{i} \int_{0}^{1} \varphi(y) y^{2 i-\mu-\nu-1} d y \quad \text { and } \quad Z_{i k}=B_{i k} \int_{0}^{1} \varphi(y) y^{2 k+\delta} d y
$$

with $A_{i}$ and $B_{i k}$ introduced previously in (16), $P$ defined in $(23), D_{\nu}(x)=$ $\left\{\begin{array}{c}B_{\nu}(x) \\ S_{\nu}(x)\end{array}\right\} f=\left\{\begin{array}{c}\cos \\ \sin \end{array}\right\} \bar{f}=\left\{\begin{array}{c}\sin \\ \cos \end{array}\right\} \delta=\left\{\begin{array}{l}0 \\ 1\end{array}\right\}$ and $M=i-1+(1-\mu-\nu)(1-\delta)$. The other parameters are read from Table V.

Some series of type (17) - (19) containing special functions instead of Bessel or Struve integrals can have similar representations in terms of Riemann zeta and related functions by a suitable choice of the function $\varphi$ in (20). We are giving two examples.

Example 1. Let us choose, for instance, $\varphi(y)=\frac{y^{1-\nu}}{\sqrt{1-y^{2}}}$. Since (see $[2$ : p. 702])

$$
\int_{0}^{1} \frac{J_{\nu}(x y) y^{1-\nu}}{\sqrt{1-y^{2}}} d y=\sqrt{\frac{\pi}{2 x}} H_{\nu-\frac{1}{2}}(x)
$$

we have $B_{\nu}(x)=\sqrt{\frac{\pi}{2 x}} H_{\nu-\frac{1}{2}}(x)$. Replacing this in (17) we get

$$
I_{1}=\sum_{n=1}^{\infty} \frac{(s)^{n-1} B_{\nu}(n x)}{n^{\alpha}}=\sqrt{\frac{\pi}{2 x}} \sum_{n=1}^{\infty} \frac{(s)^{n-1} H_{\nu-\frac{1}{2}}(n x)}{n^{\alpha+\frac{1}{2}}} .
$$

Considering that $I_{1}$ is given by (21), where $a=1, b=0, f=\cos , \delta=0$ (now $D_{\nu}=B_{\nu}$ ), we can evaluate

$$
\begin{aligned}
\sum_{n=1}^{\infty} \frac{(s)^{n-1} H_{\nu-\frac{1}{2}}(n x)}{n^{\alpha+\frac{1}{2}}}= & \frac{c \pi x^{\alpha-\frac{1}{2}}}{2^{\alpha+\frac{1}{2}} \Gamma\left(\frac{\alpha-\nu+2}{2}\right) \Gamma\left(\frac{\alpha+\nu+1}{2}\right) \cos \left(\frac{\pi(\alpha-\nu)}{2}\right)} \\
& +\sum_{i=0}^{\infty}(-1)^{i} \frac{F(\alpha-\nu-2 i) x^{\nu+2 i+\frac{1}{2}}}{2^{\nu+\frac{1}{2}+2 i} \Gamma\left(i+\frac{3}{2}\right) \Gamma(\nu+i+1)}
\end{aligned}
$$


where the other parameters we read from Table V. 702])

Example 2. Here we take $\varphi(y)=y^{\nu}\left(1-y^{2}\right)^{\nu-\frac{1}{2}}$. In this case (see [2: p.

$$
B_{\nu}(x)=2^{\nu-1} x^{-\nu} \sqrt{\pi} \Gamma\left(\nu+\frac{1}{2}\right) J_{\nu}^{2}\left(\frac{x}{2}\right) .
$$

Replacing this in (18), setting $a=1, b=0, s=-1$, we get

$$
I_{2}=\frac{2^{\nu-1} \sqrt{\pi} \Gamma\left(\nu+\frac{1}{2}\right)}{x^{\nu}} \sum_{n=1}^{\infty}(-1)^{n-1} \frac{n^{\mu-\nu}}{n^{2}-\omega^{2}} J_{\nu}^{2}\left(\frac{n x}{2}\right) .
$$

Using (22), actually formula 3 (using $\mu=-\nu$ ) from the Appendix, we evaluate the series

$$
\begin{aligned}
& \sum_{n=1}^{\infty}(-1)^{n-1} \frac{J_{\nu}^{2}\left(\frac{n x}{2}\right)}{n^{2 \nu}\left(n^{2}-\omega^{2}\right)} \\
& \quad=\frac{2^{1-\nu} x^{\nu}}{\sqrt{\pi} \Gamma\left(\nu+\frac{1}{2}\right)}\left(\frac{-\left(\frac{x}{2}\right)^{\nu}}{2 \omega^{2} \Gamma(\nu+1)} \int_{0}^{1} y^{2 \nu}\left(1-y^{2}\right)^{\nu-\frac{1}{2}} d y+\frac{\pi \omega^{-\nu-1}}{2 \sin \pi \omega} B_{\nu}(\omega x)\right) \\
& \quad=\frac{J_{\nu}^{2}\left(\frac{\omega x}{2}\right)}{2 \omega^{2 \nu+1} \sin \pi \omega}-\frac{x^{2 \nu}}{2^{4 \nu+1} \omega^{2} \Gamma^{2}(\nu+1)}
\end{aligned}
$$

\section{Appendix}

Here we list the following particular cases of (22):

$$
\begin{array}{rlr}
\sum_{n=1}^{\infty} \frac{n^{\mu} B_{\nu}(n x)}{n^{2}-\omega^{2}}= & \frac{\left(\frac{x}{2}\right)^{\nu}}{2 \omega^{2} \Gamma(\nu+1)} \int_{0}^{1} \varphi(y) y^{\nu} d y \\
& -\frac{\pi \omega^{\mu-1}}{2} \cot \pi \omega B_{\nu}(\omega x)+S_{\nu}(\omega x) \quad(\mu+\nu=0) \\
\sum_{n=1}^{\infty} \frac{n^{\mu} S_{\nu}(n x)}{n^{2}-\omega^{2}}= & \frac{\pi \omega^{\mu-1}}{2} B_{\nu}(\omega x)-\cot \pi \omega S_{\nu}(\omega x) & (\mu+\nu=1)
\end{array}
$$

for $0<x<2 \pi$,

$$
\begin{aligned}
\sum_{n=1}^{\infty}(-1)^{n-1} \frac{n^{\mu} B_{\nu}(n x)}{n^{2}-\omega^{2}}= & -\frac{\left(\frac{x}{2}\right)^{\nu}}{2 \omega^{2} \Gamma(\nu+1)} \int_{0}^{1} \varphi(y) y^{\nu} d y \\
& +\frac{\pi \omega^{\mu-1}}{2 \sin \pi \omega} B_{\nu}(\omega x) \quad(\mu+\nu=0) \\
\sum_{n=1}^{\infty}(-1)^{n-1} \frac{n^{\mu} S_{\nu}(n x)}{n^{2}-\omega^{2}}= & \frac{\pi \omega^{\mu-1}}{2 \sin \omega \pi} S_{\nu}(\omega x) \quad(\mu+\nu=1)
\end{aligned}
$$


for $-\pi<x<\pi$,

$$
\begin{array}{ll}
\sum_{n=1}^{\infty} \frac{(2 n-1)^{\mu} B_{\nu}((2 n-1) x)}{(2 n-1)^{2}-\omega^{2}}=\frac{\pi \omega^{\mu-1}}{4} \tan \frac{\pi \omega}{2} B_{\nu}(\omega x)-S_{\nu}(\omega x) & (\mu+\nu=(2) A 5) \\
\left.\sum_{n=1}^{\infty} \frac{(2 n-1)^{\mu} S_{\nu}((2 n-1) x)}{(2 n-1)^{2}-\omega^{2}}=\frac{\pi \omega^{\mu-1}}{4} B_{\nu}(\omega x)+\tan \frac{\pi \omega}{2} S_{\nu}(\omega x) \quad(\mu+\nu=1) A 6\right)
\end{array}
$$

for $0<x<\pi$, and

$$
\begin{aligned}
& \sum_{n=1}^{\infty}(-1)^{n-1} \frac{(2 n-1)^{\mu} B_{\nu}((2 n-1) x)}{(2 n-1)^{2}-\omega^{2}}=\frac{\pi \omega^{\mu-1}}{4 \cos \frac{\pi \omega}{2}} B_{\nu}(\omega x) \quad(\mu+\nu=1) \\
& \sum_{n=1}^{\infty}(-1)^{n-1} \frac{(2 n-1)^{\mu} S_{\nu}((2 n-1) x)}{(2 n-1)^{2}-\omega^{2}}=\frac{\pi \omega^{\mu-1}}{4 \cos \frac{\pi \omega}{2}} S_{\nu}(\omega x) \quad(\mu+\nu=0)
\end{aligned}
$$

for $-\frac{\pi}{2}<x<\frac{\pi}{2}$.

\section{References}

[1] Abramowitz, M. and A. Stegun: Handbook of Mathematical Functions, with Formulas, Graphs and Mathematical Tables. New York: Dover Publ. 1972.

[2] Gradshtein, I. S. and I. M. Ryzhik: Tables of Integrals, Sums, Series and Products (in Russian). Moscow: Nauka 1971.

[3] Hansen, E. R.: A Table of Series and Products. Englewood Cliffs (N.J., USA): Prentice-Hall Inc. 1975.

[4] Karatsuba, A. A.: Elements of Analytical Number Theory (in Russian). Moscow: Nauka 1983.

[5] Prudnikov, A. P., Brychov, Y. A. and O. I. Marichev: Integrals and Series. Elementary Functions (in Russian). Moscow: Nauka 1981.

[6] Prudnikov, A. P., Brychov, Y. A. and O. I. Marichev: Integrals and Series. Special Functions (in Russian). Moscow: Nauka 1983.

[7] Stanković, M. S., Djurić, M. V. and D. M. Petković: Closed form expressions for some series over product of two trigonometric functions. Facta Universitatis (Niš), Ser. Math. Inform. 9 (1994), 69 - 82.

[8] Stanković, M. S., Vidanović, M. V. and S. B. Tričković: Some series over the product of two trigonometric functions and series involving Bessel functions. Z. Anal. Anw. 20 (2001), 235 - 246.

[9] Watson, G. N.: A Treatise on the Theory of Bessel Functions. Cambridge: Cambridge Univ. Press 1958.

[10] Zaezdnyi, A. M.: Harmonic Synthesis in Radio-Technic and Electro-Connection (in Russian). Leningrad: Energiya 1972.

Received 18.05.2002; in revised form 20.09.2002 